\title{
2009 SUBJECT INDEX
}

\author{
(Volume 58, parts 1 to 4 )
}

(Covering articles, shorter articles, comments and notes, ICJ cases and current developments; page numbers in bold type indicate articles or the first page of a main entry)

Amnesties, 108

Arbitration see International Arbitration

Argentina

Emergency laws, financial crisis, 146

Armed conflict

Cluster Munitions Convention, 219

Rebellion, State responsibility, $\mathbf{4 2 7}$

Security Council authorization, 267

ASEAN Charter, 197

Asylum

Common European Asylum System, 519

Australia

Taxation, indirect, financial services, 916

Austria

Constitutional law, jurisprudence, 933

Banking

Nationalization, 119

Bilateral Investment Treaties

Compensation for expropriation, 124

Intra-EU, 297

Caribbean Court of Justice, 671

China

Trust law, 655

WTO obligations, 321

Citizenship

Fundamental rights, EU, 1002

Climate change

Bali Action Plan, 803

Financial sector, effect, $\mathbf{5 9 7}$

Cluster Munitions Convention, 219

Comparative law

Delegated legislation, 353

South Pacific, 181

Matrimonial property, 184

Suspension of performance, 867

Compensation

Bank nationalization, 124

Competition law

Cartel cases, EC, direct settlement, 627

Partnership law, $\mathbf{7 6 7}$

Complicity, by States, 1

Constitutional law

Jurisprudence, Germany and Austria, 933

Consumer Law

Collective redress, Europe, 379

Harmonization, OAS, 411

Contract

International instruments, 879

Suspension of performance, $\mathbf{8 6 3}$
Cyprus

Land rights, 1013

Denmark

Class actions, 384

Environment

Bali Action Plan, 803

Climate finance, $\mathbf{5 9 7}$

European Convention on Human Rights

Domestic law, clash with, $\mathbf{7 5 3}$

Expropriation, compensation, 128

Extraterritoriality, $\mathbf{6 8 9}$

Rape as torture, $\mathbf{5 6 5}$

Silence, right of, $\mathbf{8 3 5}$

European Court of Human Rights

International law, $\mathbf{2 6 7}$

Iraqi prisoners, $\mathbf{6 8 9}$

Silence, right of, $\mathbf{8 3 5}$

Rape as torture, $\mathbf{5 6 5}$

European Court of Justice Conflict of laws, 1013

European Union

Agriculture, 993

Asylum, harmonization, 519

Citizenship and fundamental rights, 1002

Competition law

Cartel cases, EC, direct settlement, 627

Consumer law, collective redress, 379

Free movement of goods, 985

Intra-EU bilateral investment treaties, 297

Partnership, regulatory competition, 781

Taxation, financial services, VAT, 899

Evidence International law, 163

Self-defence, standard, 168

Expropriation Compensation, bank nationalization, 124

Fair trial, right

Silence, right, 835

Family law South Pacific, 184

Fiji

Human rights, 36

Financial services

Taxation VAT, 897

Australia, 916

EU, 899

Finland

Class actions, 386 


\section{International and Comparative Law Quarterly}

France

Asylum., 526

Class actions, 389

Suspension of performance, 871

Georgia

State responsibility, secession, 493

Germany

Class actions, 393

Constitutional law, jurisprudence, 933

Delegated legislation, 353

Global warming

Climate finance, $\mathbf{5 9 7}$

Hong Kong

Refugees, non-refoulement, 443

Human rights

Indigenous peoples, 957

Security Council authorization, 267

Shareholders, 124

Silence, right of, $\mathbf{8 3 5}$

South Pacific, 31

Zimbabwe, 76

Indigenous peoples

Comparative law, South Pacific, 181

Matrimonial property, 184

UN Declaration, 957

International arbitration

Suspension of performance, $\mathbf{8 6 3}$

International Court of Justice

Current cases, 229, 713

Secessionist entities, 493

Self-defence, 163

Recommendations, 151

International Criminal Court

Zimbabwe, 112

International Criminal Tribunal for the Former Yugoslavia

Karadžić arrest and trial, 212, 726

International Tribunal for the Law of the Sea Prompt release judgments, 241

Investment treaties see Bilateral Investment Treaties

Iraq

Invasion, complicity of States, 3

Prisoner transfer, 689

Italy

Class actions, 392

Judges, 753

Judicial independence, 671

Kiribati

Human rights, 42

Kosovo

UN actions, human rights, 269
Maritime transport see Shipping

Matrimonial Property

South Pacific, 184

Nationalization Banks, UK, 119

New Zealand Human rights, 37

Norway

Class actions, 385

Organization of American States

Consumer law, harmonization, 411

Papua New Guinea

Human rights, 39

Partnership Law

Regulatory competition, $\mathbf{7 6 7}$

Portugal

Class actions, 386

Private international law

Adults, protection, 469

Companies, 1020

Consumer law, harmonization, OAS, 411

Jurisdiction agreements, 545

Common law approach, 547

Land rights, 1013

Public international law

Complicity, 1

Expropriation, compensation, 137

Necessity, 145

State immunity, $\mathbf{7 0 2}$

Refugees

Non-refoulement, Hong Kong, 443

Samoa

Human rights, 43

Sea, law of

ITLOS judgments, 241

Self-defence

Evidentiary standards, ICJ, 163

Shipping

ITLOS judgments, 241

Solomon Islands

Human rights, 41, 46

South Pacific

Comparative law, 181

Human Rights, 31

State immunity

Heads of State acting in private capacity, $\mathbf{7 0 2}$

State responsibility

Aid or Assistance, , 6

Complicity, 1

International organizations, responsibility, 281

Necessity, 145 


\section{Subject Index}

Rebellion, 427

Secessionist entities, 493

Sweden

Class actions, 383

Taxation

Financial services, VAT, 897

Tonga

Human rights, 50

Tort

Class actions, EU, 379

Torture

Rape as, $\mathbf{5 6 5}$

Transitional justice

Zimbabwe, 73

Trusts

China, 655

Agency, 659

Contractual arrangement, 659

Indivisibility of ownership, 665

Numerus Classus, 663

Separate patrimony, 657

Truth Commissions, 83

Tuvalu

Human rights, 39
United Kingdom

Asylum, 529

Banks, nationalization, 119

Class actions, 394

Delegated legislation, 353

Human rights, shareholders, 124

Iraqi prisoners, transfer, 689

United Nations

Indigenous peoples, declaration, $\mathbf{9 5 7}$

United States

Delegated legislation, $\mathbf{3 5 3}$

Partnership law, regulatory

competition, 768

Suspension of performance, 876

Vanuatu

Human rights, 51

World Trade Organization

China, accession, 321

\section{Zimbabwe}

Human rights abuse, 76

International Criminal Court, 112

Transitional justice, $\mathbf{7 3}$

Truth Commission, 98 


\title{
2009 CASE INDEX
}

\author{
(Volume 58, parts 1 to 4 )
}

7E Communications Ltd v Vertex Antennentechnik GmbH [2007] EWCA Civ 140

A v Secretary of State for Home Department (No 2) [2006] 2 AC 221

A and others v Secretary of State for the Home Department [2004] 56 (HL)

A v UK (1999) 27 EHRR 611

A et al, Abu Rideh and Ajouaou v Secretary of State for the Home Department [2004] CA 71

A-Punkt Schmuckhandels GmbH v Claudia Schmidt Case C-441/04 [2006] ECR I-2093

Abbot Laboratories v Gardner (1967) 387 US 136

Abdulaziz v UK (1985) 7 EHRR 471

Abdulla, Aydin Salahadin v Federal Republic of Germany, ECJ C-175-179/08 (pending) 524

Accenture, C-472/03 [2005] ECR I-1719

ACCC v J McPhee (1998) ATPR 41-628

ACCC v NW Frozen Foods Pty Ltd (1996) ATPR 41-515 42, 441

913

648

648

Accord ACSYNGO v. Compagnie de Saint-Gobain (France) SA, 82 ILR 127, (Belgium Comm. Ct. 1986)

Activities on the Territory of the Congo (DRC v Rwanda) [2002] ICJ Reports 249

AF (NZ) v Zaoui [2005] NZSC 38

462

Affaire du Lac Lanoux (Spain v France) (1957), 12 RIAA $28 \quad 12$

AIG Europe (UK) v The Ethniki [2000] 2 All ER 566 550

Akhtar v Secretary of State [2001] SLT 1239

Akkoc v Turkey (2002) 34 EHRR 51 581

Åklagaren v Mickelsson, Roos, Case C-142/05 987

Aksoy v Turkey [1996] ECHR 68 567

Al Adsani v UK (2002) 34 EHRR 11

Alama v Tevasa [1987] SPLR 385

Alexandria v US (1984) 737 F 2d 1002 (Fed. Cir.) 367

Allan v UK (2003) 36 EHRR 12

AM \& S Europe Ltd v Commission ECJ Case 155/79

American International Group, Inc. v Islamic Republic of Iran Award No 93-2-3, 4 Iran-US CTR 96, 106 (19.12.83)

$\begin{array}{ll}\text { American Textile Manufacturers Institute v Donovan (1981) 452 US } 490 & 359 \\ \text { American Trucking Assn v Atchison (1967) } 387 \text { US } 397 & 359\end{array}$

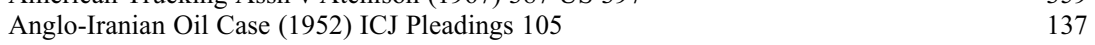

Anheuser-Busch v Portugal (2007) 45 EHRR 36

Aoulmi v France 2006-I, 46 EHRR 1 710

Apostolides v Orams [2006] EWHC 2226 (QB); [2007] 1 WLR 241

Apostolides v Orams, Case C-420/07, ECJ, 28.4.09 1013

Appleton v Appleton [1965] 1 WLR 25 187

Application of the Convention on the Prevention and Punishment of the Crime of Genocide (Bosnia and Herzegovina v Serbia and Montenegro) 5, 168, 215, 281, 427, 495, 720

Application of the Convention on the Prevention and Punishment of the Crime of Genocide (Croatia v Serbia)

Application of the International Convention on the Elimination of All Forms of Racial Discrimination (Georgia v Russia) (ICJ)

Archer Daniels Midland v Commission, ECJ Case C-397/03P, Judgment 18.5.06 633

Archer Daniels Midland v Commission, Case T-224/00, [2003] ECR II-2597 633

Argentina-Footwear, WTO Appellate Body Report WT/DS121/AB/R

Armed Activities in and Against Nicaragua (Nicaragua v USA) (Judgment, Merits) (1986) [1986] ICJ Rep 14 


\section{International and Comparative Law Quarterly}

Armed Activities on the Territory of the Congo (DRC v Uganda) [2005]

ICJ Reports 158

$151,163,174,495$

Asahi Metal Industry, (1987) 480 US 102

Ashby v Minister of Immigration [1981] 1 NZLR 223

Ashby, Glen v Trinidad and Tobago, 26.7.94, UNHRC Communication No. 580/1994
Association et al v State of Netherlands and United Nations (Judgment in Incidental Proceedings) (7.10.08)

Association of Data Processing Service Organizations, Inc. v Camp (1970) 197 US 150

Assurador-Societetet, C-8/01, [2003] ECR I-13711

Asylum Case ICJ Reports 1950, 266

AG (Fiji) v Silatolu, CA, Fiji, 6.3.03, [2003] FJCA 12

AG (Ireland) v Jameson [1905] 2 IR 218

AG Superintendent of Prisons Chief Marshal v Jeffrey Joseph Lennox and Ricardo Boyce, CCJ Appeal No CV 2 of 2005

AG's Reference (No 2 of 2001)

Australian Conservation Foundation v. Minister for Planning, [2004] VCAT 2029, 23

Aydin v Turkey (1998) 25 EHRR 251

Azapo v President of the Republic of South Africa 1996 (4) SA 671 (CC)

Bahadur v Secretary for Security [2000] 2 HKLRD 113

Baker v Canada [1999] 2 SCR 817

Baker v R [1975] AC 774

Banco Nacional de Cuba v Sabbatinoi (1964) 376 US 398

Banga v Waiwo [1996] VUSC 5

Banković v Belgium, ECtHR App No 52207/99, 11 BHRC 435

Barrios Altos (IACHR, Vol. 75 (Series C), 27.11.98)

BASF and UCB v Commission, ECJ Joined Cases T-101/05 and T-111/05, OJ [2008] $\mathrm{C} 22 / 41$

Bates v Lord Hailsham (1972) 1 W.L.R. 1373

Batterton v Marshall (1980) 648 F 2d, 694 (DC Cir)

Baumbast and R v Secretary of State for the Home Department Joined Cases C-413/99 [2002] ECR I-7091

Baumgarter v Baumgarter [1987] 164 CLR 137

Bayindir Insaat Turizm Ticaret Ve Sanayi AS v Pakistan Decision on Jurisdiction, ICSID Case No ARB/03/29 (14.11.05)

Behrami and Behrami v France, ECtHR Appl. No 71412/01, 2.5.07

Behrami and Saramati v France, Germany and Norway Appl. No 71412/01, and Appl. No 78166/01 (2.5.07)

Benincasa v Dentalkit Srl, Case C-169/95, [1997] ECR I-3767

$235,267,508$

Bensusan Restaurant Corp v King, 937 F Supp 295 (SDNY 1996)

Berghoefer GmbH \& Co v ASA SA, Case 221/84, [1985] ECR 2699

Beric v Bosnia and Herzegovina, ECtHR App Nos 36357/04 (etc), 16.10.07

Bidar C-209/03 [2005] ECR I-2119

Biloune and Marine Drive Complex Ltd v Ghana Investments Centre and the Government of Ghana, Award on Jurisdiction and Liability (27.10.89) 95 ILR 183

Biwater Gauff (Tanzania) Ltd (UK) v United Republic of Tanzania, ICSID ARB/05/22, Award (24.7.08)

Bols Distilleries v Superior Yacht Services [2006] UKPC 45, [2007] 1 WLR 12

Bosphorus Hava Yollari Turizm ve Ticaret AS v Minister for Transport, Energy and Communications and others C- 84/95 [1996] ECR I-3953

Bosphorus Hava Yollari Turizm ve Ticaret ASi v. Ireland, 2005, (ECtHR Appl. No. 45036/98)

Boumediene v Bush, slip opinion, 553 US (2008) 25

Bowen v American Hospital Association (1986) 476 US 610

Bowen v Georgetown University Hospital (1988) 488 US 204

Brennan v UK (2002) 34 EHRR 18

Brian Curran v the State; Minister for Foreign Affairs; Arnold Marsupial, Bernard Naracoopa and Lucas Wake, as Members of a Ministerial Committee of Review [1994] PNGLR 230 


\section{Case Index}

Brown v Government of Rwanda and the Secretary of State for the Home Department [2009] EWHC 770 (Admin)

Brown v Stott [2001] 2 WLR 817

W Bulthuis-Griffioen, Case C-453/93 [1995] ECR I-2341

Burger King v Rudzewicz (1985) 471 US 462

Burrardview Neighbourhood Association v Vancouver [2007] 2 SCR 86

C v Director of Immigration [2008] 2 HKC 167

C Inc v Australian Crime Commission [2008] FCA 1806

Cabvision Ltd v Feetum \& Marsden (Feetum v Levy) [2005] 1 WLR 2576

Cahuas, Olaechea v Spain ECHR 2006-X

Calder v Jones (1984) 465 US 783

Camouco (Panama v France) (Prompt Release, Judgment of 7.2.00) ITLOS Reports 200010

Campbell v MGN Ltd [2004] UKHL 22

705

840

901

480

461

443

453

784

481

244

33

547

558

Carnoustie Universal SA v International Transport [2002] 2 All ER (Comm) 657

Cartesio Oktatóés Szolgáltatóbt, ECJ Case C-210/06, 16.12.08

Cartonboard [1998] ECR II-2099

Case Concerning Sections 301-310 of the Trade Act of 1974 (EU v USA 1999)

Centros Lt. v Erhvervs-og Selskabsstyrelsen, ECJ Case C-212/97, [1999] ECR I-1459

Certain Criminal Proceedings in France (Republic of the Congo v France) ICJ Rep 2003

Certain Norwegian Loans (France v Norway) (Merits) [1957] ICJ Rep 9

Chagos Islanders v Attorney-General [2003] EWHC 2222, leave to appeal refused: [2004] EWCA Civ 997

Chahal v UK (1996) 23 EHRR 413

Chan Mei Yee v Director of Immigration (unreported, HK CFI, 13.7.00)

Chan To Foon v Director of Immigration [2001] 3 HKLRD 109

Channel Tunnel Group Ltd v Balfour Beatty Contruction Ltd [1992] Q.B. 656

Chen Case C-200/02 [2004] ECR I-

Chow Hung Ching v R (1949) 77 CLR 449

Cheffers v Cheffers [1993] HBSC 2

Chiu v Minister for Immigration [1994] 2 NZLR 541

Chocosuisse Union des Fabricants Suisse de Chocolat v Cadbury Ltd [1998] ETMR 205

Chu Woan Chyi v Director of Immigration [2007] 3 HKC 168

Chung Chi Cheung v R [1939] AC 160

CME Czech Republic BV (Netherlands) v Czech Republic, Final Award (14.3.03)

CMS Gas Transmission Co v Argentina, ICSID Case No ARB/01/8, Award (12.5.05)

Commission v France, ECJ C-76/99, [2001]ECR I-249

Commission v Ireland, ECJ, Case C-459/03, 30.5.06

Commission v Italy, ECJ Case 10/61, 27.2.62

Commission v Italy, Case 122/87, [1988] ECR 2685

Commission v Italy, Case C-110/05, 10.2.09

Commission v Portugal, Case C-265/06, [2008] ECR I-2245

Commission v SGL Carbon, ECJ Case C-301/04 P, OJ [2006] C 224, 29.6.04

Commission v Spain, ECJ C-124/96, [1998] ECR I-2501

Commission v UK, ECJ Case 253/85 [1988] ECR 817

Commissioner of Rating and Valuation v Agrila Ltd [2001] 2 HKLRD 36

Commodity Futures Trading Commission v Schor (1986) 478 US 833

Community Nutrition Institute v Young (1987) 818 F 2d, 943, 951 (DC Cir)

Compagnie de Saint-Gobain Zweigniederlassung Deutschland v Finanzamt Aachen-Innenstadt, ECJ C-307/97, 21.9.99

Compaňia del Desarrollo de Santa Elena SA v Costa Rica, ICSID Case No ARB/96/1, Award (17.2.00)

Compare International Trader Ltd v Town Planning Appeal Board (unreported, HK CFI, A Cheung J, 15.11.07)
793, 1021

649

831

793,1022

721

173

464

763

453

453

873

1002

459

184

453

396

463

451

127

143

902

318

306

901

987

988

637

902

901

463

359

371

309

127

467 


\section{International and Comparative Law Quarterly}

Competence of the General Assembly for the Admission of a State to the UN [1950] ICJ Rep 4

Comunidad Indigena Yakye Axa v Paraguay, IACHR, Series C 125 (2005) 974

I Congresso del Partido [1983] 1 AC 244

Consolidated Coal Co. v Illinois (1902) 185 US, 203

Constitution of the Republic of Vanuatu and the Infant P and her Natural Mother S [1980-88] 1 VLR 130

Continental Shelf Case, ICJ, (Libya/Malta)

Continental Shelf Case (Tunisia/Libya) (Merits) [1982] ICJ Rep 18

Cookies World, C-155/01, [2003] ECR I-8785

Coreck Maritime GmbH v Handelsveem, ECJ Case C-387/98 [2000] ECR 9337

Corfu Channel (UK v Albania) (Merits) [1949] ICJ Rep 4

Costello-Roberts v UK [1993] ECHR 16

Coy et al v Attorney General of Belize et al, Supreme Court of Belize, Claims No 171 and 172, 10.10.07

CPP, C-349/96, [1999] ECR I-973

Credit Suisse Financial Products v Société Generale d'Enterprises [1997] CLC 168 (CA) 551

Cruz Varas v Sweden (1991) 14 EHRR 1

CSC Financial Services, C-235/00, [2001] ECR I-10237

Cundy v Lindsay (1878) 3 App Cas 459

Curran, Brian v the State; Minister for Foreign Affairs; Arnold Marsupial, Bernard Naracoopa and Lucas Wake, as Members of a Ministerial Committee of Review [1994] PNGLR 230

Custom Made Commercial Limited v Stawa Metallbau, ECJ Case C-288/92, [1994] ECR 2913

Cybersell, Inc v Cybersell, Inc, 130 F.3d 414 (9th Cir 1997)

$\mathrm{D} v$ Inspecteur van de Belastingdienst/Particulieren/Ondernemingen buitenland te Heerlen, ECJ C-376/03, 5.7.05

D v UK [1997] 24 EHRR 423

Daily Mail and General Trust, ECJ Case 81/87, [1988] ECR 5483

D’Ambrumenil, ECJ C-307/01, [2003] ECR I-13989 902

Dansk Rørindustri A/S v Commission of the European Communities, ECJ Case C-189/02 P, OJ [2002] L 202

De Plessis v De Klerk (1996) BCLR 658

Delimitation of the Maritime Boundary in the Gulf of Maine Area (Canada v USA) (Judgment) (1984) ICJ Rep 246

Dell Computer Corp v Union des consommateurs et Olivier Dumoulin (2007 CSC 34), 13.7.07

Denizci and others v Cyprus [2001] ECHR 351

Denuit, Guy, ECJ C125-/04, 27.1.05

Deutsche Bank v Asia Pacific Broadband Wireless [2008] EWCA 1091

Devi v Singh [1985] FJCA 2

Deweer v Belgium (1980) 2 EHRR 439

Dikme v Turkey [2000] ECHR 366 


\section{Case Index}

E v UK, Appl. no 33218/96, 26.11.02

Eastern Sugar BV v Czech Republic (ad hoc arbitration under UNCITRAL rules), Partial Award, 27.3.07; Final Award 12.4.07

EC-Bananas, WTO Panel Report, WT/DS27/R

EC-Hormones-Report of the Appellate Body WT/DS26/AB/R WT/DS48/AB/R 347

Eckle v Federal Republic of Germany (1983) 5 EHRR 1

Eco Swiss China Time Ltd v Benetton International NV, ECJ C-126/97, 1.6.99 301

EEA Agreement, ECJ Opinion 1/91, 14.12.91 308

Egmez v Cyprus (2002) 34 EHRR 29

Elefanten Schuh v Pierre Jacqmain, ECJ Case 150/80, [1981] ECR 1671

Elettronica Sicula S.P.A. (ELSI) (USA v Italy) (Merits) [1989] ICJ Rep 15

Elgafaji ECJ Case C-465/07 525

Elide Gottardo v Istituto nazionale della previdenza sociale (INPS), ECJ C-55/00, 15.1 .02

Elisara v Elisara [1994] WSCC 14

EM (Lebanon) v Secretary of State for the Home Department, [2006] EWCA Civ 1531, [2008] 3 WLR 931 (HL)

Enron v Argentina, ICSID Case No. ARB/01/3, Award (22.5.07)

Eridana, ECJ C-230/78, [1979] ECR 2749

Eritrea/Ethiopia Claims Commission, Partial Award, Jus ad Bellum (Ethiopia claims 1-8)

Exchange of Greek and Turkish Populations (Advisory Opinion) PCIJ Rep Series B No 10

Exportur SA v LOR SA and Confiserie du Tech SA, ECJ Case 3/91, 10.11 .92

F v UK Appl. no. 17341/03, 22.6.04

Fabbrica Italiana Accumulatori Motocarri Montecchio SpA (FIAMM) and Others v Council and Commission, Joined Cases C-120/06 P and C-121/06 P, 9.9.08 742

Fay v Noia, 372 U.S. 391, 438 (1963)

FB v Director of Immigration (unreported, Hong Kong Court of First Instance, Saunders J, 5.12.08

FB v Director of Immigration [2009] 1 HKC 133

Federal Power Commission v New England Power Co (1974) 415 US 352

Fernandes v Secretary of State for Home Department [1981] Imm A R 1

Ferreira v Levin 1996 (1) BCLR 1

Fertilizer Corporation of India case (1984) 2 ICSID Rev 19

453

FG Hemisphere Associates LLC v Democratic Republic of Congo (unreported, Hong Kong Court of First Instance, Reyes J, 12.12.08 459

Field v Clark (1892) 143 US, 649

Fiona Trust \& Holding Corporation v Privalov [2007] EWCA Civ 20 (CA) [2007] UKHL 40 (HL)

Fisheries Jurisdiction Case (Federal Republic of Germany v Iceland) (Judgment, Merits) [1974] ICJ Rep 175

Fisheries Jurisdiction Case (UK v Iceland) (Judgment, Merits) (1974) [1974] ICJ Rep 3

Fisheries Case (UK v Norway), (Judgment) (1951) [1951] ICJ Rep 1951, 116

Fornah v Secretary of State for the Home Department [2006] 46 (HL)

Francis v Henderson, 425 U.S. 536, 539 (1976)

Free Zones of Upper Savoy and the District of Gex, P.C.I.J., Series A, No. 22

Friends of the Earth $\mathrm{v}$ The Governor in Council and the Minister of the Environment, [2008] FC 1183

Frontier Dispute, I.C.J. Reports 1986 


\section{International and Comparative Law Quarterly}

Galeries Segoura SPRL v Firma Rahim Bonakdarian, ECJ Case 25/76, [1976] ECR 1851

Garza, Juan Raul v USA, Decision on the Merits, 4.4.01 IA Comm. HR, Case No 12.243, Report No 52/01

Gemeente Leusden and Holin Groep Joint Cases C-487/01 and C-7/02, [2004] ECR I-5337

Generation Ukraine, Inc v Ukraine, ICSID Case No ARB/00/9, Award (16.9.03)

Germany v Commission, ECJ C-240/90 [1992] ECR I-5383

Germany v Parliament and Council (Tobacco Advertising) Case C-376/98 [2000] ECR I-8419

Gillies v Keogh [1989] 2 NZLR 327

Gissing v Gissing [1971] AC 886

Grand Prince (Belize v France) (Prompt Release, Judgment of 20.4.01) ITLOS Reports 200117

Grant v Edwards [1986] 2 All ER 426

Graphite Electrodes-Commission Decision of 18.7.01 (Case 36.490) OJ [2002] L 10070

Greek case, the, [1969] 12 YB ECHR 1

651

580

902

Gregg, ECJ C-216/97, [1999] ECR I-4947

Grimaud v US (1911) 220 US, 506

358

987

Gryzelczyk Case C-184/99 [2001] ECR I-6193

1002

Guatemala-Cement, WTO Appellate Body Report WT/DS60/AB/R 325

Gudmundsson v Iceland (1960) YB ECHR 394, (E. Comm. HR) 129

Gysbrechts, Lodewijk, ECJ Case C-205/07, 16.12.08 988

Halapua v Tonga [2004] TOCA 5 185

Halina Nerkowska v Zakład Ubezpieczen’ Społecznych ECJ C-499/06 22.5.08 1002

J.W. Hampton Co. v US (1928) 276 US, 394

Hanover Shoe v United Shoe Machinery Corp 392 US 481 (1968)

Hanson v Denckla, (1958)357 US 235

Harrikissoon v Attorney-General of Trinidad and Tobago [1980] AC 265

358

644

480

Helnan International Hotels A/S v Arab Republic of Egypt, ICSID Case No ARB/05/19, Award (3.7.08)

Henkel ECJ C-167/00 [2002] I-8111

Hilaire et al v Trinidad \& Tobago, Judgment, 21.6.02, IACHR (Series C) No 94 (2002) 709

Hoffman, ECJ C-144/00, [2003] ECR I-2921 902

Hong Kong Fir Shipping Co v Kawasaki Kisen Kaisha [1962] 2 QB $26 \quad 875$

HKSAR v Hung Chan Wa [2006] 3 HKLRD 841

HKSAR v Yav Mee Kwan [2004] 1 HKLRD A6 463

Horvath v Secretary of State for the Home Department [2001] 1 AC 489

Hoshinmaru (Japan v Russian Federation) (Prompt Release, Judgment of 6.8.07)
www.itlos.org/case documents/2007/document en 295.pdf

Howarth v UK (2000) 31 EHRR 861

Humphrey's Executor v US (1935) 295 US 602

Hutchinson v Newbury Magistrates' Court [2000] EWHC QB 61 459

Ilascu v Moldova and Russia, ECtHR App No 48787/99, GC, 8.7.04 271, 508

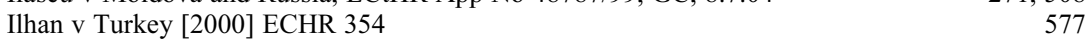

Illinois Brick Co v Illinois 431 US 720 (1977)

Industrial Department AFL-CIO v American Petroleum Institute (1980) 448 US $607 \quad 359$

Inglis v Loh Lai Kuen Eda [2005] 3 HKC 115

Institute of Motor Industry, ECJ C-149/97, [1998] ECR I-7053 902

International Shoe v Washington, 326 US 310 (1945) 480

Internationale Handelsgesellschaft [1970] ECR $1125 \quad 233,1011$ 


\section{Case Index}

IP Metal v Route OZ SpA [1994] 2 Lloyd's Rep 560

Ireland v UK (1979-80) 2 EHRR 25

Islam v Secretary of State for the Home Department [1999] 2 WLR 1015

Island of Palmas Case (Netherlands v USA) (1928), 2 RIAA, 82 21

Italia Ta'amale and Ta'amale Toelau v The Attorney-General (CA,Samoa, 18.8.95) 57

Iveco Fiat SpA v Van Hool, ECJ Case C 313/85, [1986] ECR 3337

Jacob and Youngs v Kent, 230 NY 239, 129 N.E. 889 (1921) 878

Jalloh v Germany (2007) 44 EHRR $32 \quad 836$

James v UK (1998) Series A No 98, 8 EHRR 123

James et al v Trinidad and Tobago, Order of 3.4.09, IACHR (unpublished) 709

Januzi and Hamid v Secretary of State for the Home Department [2006] UKHL 5

Japan-Alcoholic, WTO Appellate Body Report WT/DS8/AB/R 332

JB v Switzerland ECtHR Appl. 31827/96 $\quad 842$

JP Morgan, Case C-363/05 [2007] ECR I-5517 903

JFE Engineering Corp v Commission, ECJ Joined Cases T-67/00 T-68/00, T-74/00 and
T-78/00, 8.7.04

Jia Case C-1/05 [2007] ECR I-1 1002

Johnson, Doreen v Caribbean Centre for Development Administration [2009] CCJ 3 (OJ)

Joli v Joli [2003] VUSC 63, [2003] VUCA 27, [2004] VUSC 91

Jones v Saudi Arabia [2007] 1 A.C. 270

JPS BVBA v Kabri Mode BV, AR 3641/94, Rechtbank van Koophandel 1.3.95 (District Commercial Court, Hasselt, Belgium, < http://www. unilex.info > 883

Juno Trader (Saint Vincent and the Grenadines v Guinea-Bissau) (Prompt Release, Judgment of 18.12.04) ITLOS Reports 200417

K v Austria ECtHR Series A no 255-B, 2.6.93

841

Kadi, Yassin Abdullah and Al Barakaat International Foundation v Council and Commission ECJ Joined Cases C-402/05 and C-415/05 P, Judgment, 3.9.08229,

Kadi, Yassin Abdullah and Al Barakaat International Foundation v Council and Commission T-315/01, [2005] ECR II-3649

Kalotano, Family v Duruaki Council of Chiefs (SC, Vanuatu, 24.5.02), [2002] VUSC 32

Kamer van Koophandel en Fabrieken voor Amsterdam v Inspire Art Ltd, Case C-167/01 ECR [2003] I-10155

Kasumaj v Greece, ECtHR App No 6974/05, 5.7.07

793,1023

295

581

$\begin{array}{ll}\text { KB v National Health Service Pensions Agency ECJ Case C-117/01 } & 526 \\ \text { Keck and Mithouard Cases C-267 and C-268/91 [1993] ECR I-6097 } & 986\end{array}$

$\begin{array}{ll}\text { KB v National Health Service Pensions Agency ECJ Case C-117/01 } & 526 \\ \text { Keck and Mithouard Cases C-267 and C-268/91 [1993] ECR I-6097 } & 986\end{array}$

Khosrowshahi v Islamic Republic of Iran, Award No. 558-178-2, 30
Iran-U.S.C.T.R. 76, 92 (1994)

Kingscrest Associates and Montecello, C-498/03, [2005] ECR I-4427 902

Kingston v Preston [1773] 99 Eng Rep 437 (KB) 872

Klöckner Industrie-Anlagen GmbH (FR Germany) v United Republic of Cameroon (1994) 2 ICSID Rep 95; (1986) XI Ybk Commercial Arbitration 162 (annulment decision) 887

Klomps v Michel, Case 166/80, [1981] ECR $1593 \quad 1019$

Kluger, Case C-141/00, [2002] ECR I-6833 902

Kong v Kong [2000] VUCA $8 \quad 185$

Koon Wing Yee v Insider Dealing Tribunal [2008] 3 HKLRD 372

Korea-Dairy, WTO Appellate Body Report WT/DS98/AB/R 325

Koroiwaca v Bakosa [2004] FJHC 207 184

Kōster, C-25/70 [1970] ECR, 1161 358

Kostovski v Netherlands (1991) 12 EHRR 434

Krennemer Golf, C-174/00, [2002] ECR I-3293 902

Krombach v Bamberski C-7/98, [2000] ECR I-1395 406

Kronhofer Case C-168/02 [2004] ECR I-6009 479

Kutner v Phillips [1891] 2 QB 267

$\begin{array}{ll}\text { L v L [1994] WSCA 3 } & 187\end{array}$ 


\section{International and Comparative Law Quarterly}

Lafaialii v AG and the Alii \& Faipule of Falealupo (SC, Samoa, 24.4.03), [2003] WSSC 8

LAFI Office and International Business SL v Meriden Animal Health Ltd [2001] ILPr 237

LaGrand (Germany v USA) ICJ

Lai Tak Shing v Director of Home Affairs (unreported, HKCFA,

CACV 201/2005, 9.10.06) and (unreported, HKCFA, FACV 5/2007, 5.11.07)

Land and Maritime Boundary between Cameroon and Nigeria Provisional Measures, ICJ Rep 1996

$\begin{array}{lr}\text { Land Rheinland-Pfalz v Alcan Deutschland GmbH, ECJ C-24/95, 20.3.97 } & 317 \\ \text { Lange v Atkinson [1997] 2 NZLR 22; CA (1998) 4 BHRC 573 } & 38\end{array}$

Lau Kong Yung v Director of Immigration [1999] 3 HKLRD 778

Laval Case C-438/05 [2007] ECR I-10779 989

Le Tu Phuong v Director of Immigration [1994] 2 HKLR 212

Lee Bun v Director of Immigration [1990] 2 HKLR 466

Legality of the Use of Force (Yugoslavia v Belgium) [1999] ICJ Reports 132

Legality of the Use by a State of Nuclear Weapons in Armed Conflict [1996-I] ICJ Reports

Leung Kwok Hung v President of Legislative Council [2007] 1 HKLRD 387

Lewis v Avery [1972] 1 QB 198 (HL)

LG\&E Energy Corp v Argentina, ICSID Case No ARB/02/1, Decision on Liability (3.10.06)

Lichter v US (1948) 334 US 742

Liggett v Lee, 288 US 517

Lindqvist ECJ Case C-101/01

Lisresal [1996] (ECJ C-32/95 P) ECR I-5373

Lithgow (Sir William) v UK, Series A 102 (1986), 8 EHRR 329

Littrell v USA (No 2) [1995] 1 WLR 82

Lloyds Bank v Rosset [1990] 1 All ER 111

Lockyer v General Motors No 3:06. Civ 05755 (ND California, filed 20.9.06)

Loizidou v Turkey ECtHR Appl.No 15318/89, (1997) 23 EHRR

S.S. Lotus (France v Turkey) (Judgment) (1927) Series A, No. 10

Loumia v DPP [1985/6] SILR 158

Luu The Truoung v Chairman of the Refugee Status Review Board [2003]

$$
2 \text { HKLRD } 351
$$

MA (Palestinian Territories) v Secretary of State for the Home Department, CA, 9.4.08 744

Madaha Resena v PNG [1991] PNGLR $174 \quad 58$

Mayagna (Sumo) Awas Tingni Community v Nicaragua, IACHR, Series C 79 (2001) 980

Magee v UK (2001) 31 EHRR 35

Maharaj v AG for Trinidad and Tobago (No 2), [1979] AC 385; [1981] AC 61

Mamac v Turkey, ECtHR Appls. Nos 29486/95, 29487/95, 29853/96, 20.4.04 858

$\begin{array}{lr}\text { Mamatkulov and Askarov v Turkey (2005) } 41 \text { EHRR } 494 & 709\end{array}$

Manning v Hill (1995) 126 DLR (4th) 129

Marshall-Barnett v AG of Jamaica PC Appeal No 41 of 2004

Matteucci v Communauté francaise of Belgium and Commissariat général aux relations
internationales of the Communauté francaise of Belgium, ECJ C-235/87 306

Mauga and Others v Leituala (Unreported, CA, Samoa, March 2005) 41

Mavrommatis Palestine Concessions, (Greece v UK) PCIJ Series A, No. $2 \quad$ 152, 254, 718

MC v Bulgaria [2003] ECHR 646 566

Medical Council of Hong Kong v Chow Siu Shek [2000] 2 HKLRD 674

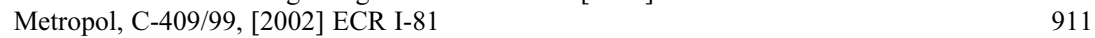

Midland Bank, C-98/98 [2000] ECR I-4177 908

Migrationsverket v Edgar Petrosian and Others, ECJ C-19/08 524

Military and Paramilitary Activities in and against Nicaragua, Case Concerning, (Nicaragua v USA) (Merits) [1986] ICJ Rep $14 \quad 137,155,164$, 454, 496 


\section{Case Index}

Miller v Jackson [1977] QB 966

1030

Mines de potasse d'Alsace ECJ Case 21/76 [1976] ECR 477

Minister of Home Affairs v Fisher [1980] AC 319

Minquiers and Ecrehos Case (France v UK) (Judgment), [1953] ICJ Rep 47

Mistretta v US (1989) 488 US 361

Moc A Pao v Director of Immigration (unreported, Supreme Court of Hong Kong, 19.5.97)

Moiwana Community v Suriname, IACHR, Series C 124 (2005) 980

Moldovan v Romania [2005] ECHR $458 \quad 570$

Molu v Molu [1998] VUSC 15 185

Mondev International Ltd v USA, ICSID Case No ARB(AF) 99/2, Award (11.10.02) 135

Monte Confurco (Seychelles v France) (Prompt Release, Judgment of 18.12.00) ITLOS Reports 200080

MOX Plant case (Ireland v UK)

242

301

551

128

$\begin{array}{ll}\text { MTD Equity Sdn Bhd v Chile, ICSID Case No ARB/01/7, Award (25.5.04) } & 128 \\ \text { Munaf v Geren, Slip opinion, 553 US (2008) 17-21 } & 701\end{array}$

Munar v Canada [2005] FC 1180

Murdoch v Murdoch [1975] 1 SCR 423

Murray, John v UK (1996) 22 EHRR 29

467

837

M/V Saiga (Saint Vincent and the Grenadines v Guinea) (Prompt Release, Judgment of 4.12.97) ITLOS Reports 199710

M/V Saiga (No. 2) (Saint Vincent and the Grenadines v Guinea) (Merits, Judgment of 1.7.1999) ITLOS Reports $199910 \quad 247$

Murphy v Waterfront Commission (1964) 378 US 52

Muschinski v Dodds [1985] 160 CLR 583 186

N v Secretary of State for the Home Department [2005] 2 AC $296 \quad 760$

NAACP v Federal Power Commission (1976) 425 US 662

Nagol Jump, Assal and Vatu v Council of Chiefs of Santo [1989-1994] 2 VanLR 545

Nasseri, Javad v The Secretary of State of the Home Department, [2007] EWHC 1548 (Admin), [2009] 2 WLR 523

Nationale Raad van Dierenkwekers en Liefhebber, C-219/07 [2008] ECR I-4475 988

Ng Siu Tung v Director of Immigration [2002] 1 HKLRD 561 464

Ngo Thi Minh Huong (An Infant) v Director of Immigration (2001) 9 HKPLR $186 \quad 444$

Nguyen Tuan Cuong v Director of Immigration [1997] 1 WLR $68 \quad 444$

Noel, John v Obed Toto (Unreported, SC, Santo, Vanuatu, 1998) 51

Nordsee Deutsche Hochseefischerei GmbH, ECJ C-102/81, 23.3.82 301

North Carolina v Alford, 400 US 25 (1970) 631

North Sea Continental Shelf Cases (Federal Republic of Germany v. Denmark; Federal Republic of Germany v Netherlands) (Judgment) (1969) [1969] ICJ Rep 3

Nuclear Tests Case (Australia v France) [1974] ICJ Rep 253

$24,135,433$

831

650

459

Nulyarimma v Thompson (1999) 165 ALR 621

O'Halloran and Francis v UK Application nos. 15809/02 and 25624/02, 29.6.07

836

858

301

Occidental Exploration v Ecuador, CA, 9.9.05

460

Odelola v Secretary of State for Home Department [2008] EWCA Civ 308

Oil Platforms (Islamic Republic of Iran v USA) (Merits) [2003] ICJ Rep 161

Okoloubu v Canada [2007] FC 1069

Oliver v Bradley [1987] 1 NZLR 586

Open Skies, ECJ C-466-469/98 C-471-472/98, C-475-476/98, 5/11/02

Orkem v European Commission, ECJ Case 374/87, [1989] ECR 3283

Owusu v Jackson, ECJ Case C-281/02, [2005] ECR I-1383

Pafco Employees Union v Pacific Fishing Company Ltd [2002] ICHRL 4; (2003) 4 CHRLD 6

165,308

467

186

313

742,835

1013

Pale v Pohiva [2006] TOSC 16

Panama Refining Co. v Ryan (1934) 293 US, 388 


\section{International and Comparative Law Quarterly}

Panovits v Cyprus, Appl. No 4268/04, 11.12.08 $\quad 859$

Parliament v Council, ECJ C-417/93, [1995] ECR, I-1185 358

Parliament v Council, ECJ C-303/94, [1996] ECR, I-2943 358

Partenreederei M/S Tilly Russ v Haven \& Vervoerbedrijf Nova, ECJ Case C-71/83,

$\begin{array}{ll}\text { [1984] ECR } 2417 & 559 \\ & \end{array}$

$\begin{array}{ll}\text { Parti écologiste 'Les Verts' v European Parliament, ECJ, C-294/83, 23.4.86 } & 318 \\ \text { Pasi v Kamana [1986] 1 NZLR 603 } & 186\end{array}$

Passage through the Great Belt (Finland v Denmark) [1991] ICJ Reports $20 \quad 154,721$

PCCW-HKT Telephone Ltd v Telecommunications Authority [2005] 3 HKLRD $235 \quad 463$

Pettit v Pettit [1970] AC 777 186

Pettkus v Becker [1980] 2 SCR 834 186

Plante v Jacobs, 10 Wis. 2d 567, 103 N.W. 2d 296 (1960) 878

Dante Piandiong, Jesus Morallos and Archie Bulan v the Philippines, 19.10.00,
UNHRC Communication No 869/1999

Pohiva v Prime Minister and Kingdom of Tonga [1988] LRC (Const) $949 \quad 50$

Police v O'Connor [1992] 1 NZLR 87

Powell Duffryn Plc v Wolfgang Petereit, ECJ Case C-214/89,[1992] ELR 1745

Prabakar v Secretary for Security (unreported, Hong Kong Court of Appeal,
CACV 211/2002, 27.11.02) \begin{tabular}{lr} 
CACV 211/2002, 27.11.02) & 449 \\
\hline
\end{tabular}

Premalal v Minister for Immigration, Local Government and Ethnic Affairs (1993) $\begin{array}{ll}41 \text { FCR } 117 & 452 \\ & 535\end{array}$

$\begin{array}{ll}\text { Pretty v United Kingdom ECtHR Appl. No 2346/02 29.4.02 } & 535 \\ \text { Preussen Elektra, C-379/98 [2001] ECR I-2099 } & 989\end{array}$

Process Gas Consumers Group v Consumer Energy Council of America (1983)
463 US 1216 $\begin{array}{ll}463 \text { US } 1216 & 366 \\ & 566\end{array}$

$\begin{array}{ll}\text { Prosecutor v Akayesu, Case no ICTR-96-4-T, 2.9.98 } & 566 \\ \text { Prosecutor v Barayagwiza, ICTR-97-19, (Judgment) (3 November 1999) } & 740\end{array}$

$\begin{array}{ll}\text { Prosecutor v Blaskić ICTY-95-14-T } & 513,721\end{array}$

Prosecutor v Delalić ICTY-96-21-A $\quad 506$

Prosecutor v Thomas Lubanga Dyilo ICC-01/04/06 515

Prosecutor v Furundzija (ICTY) IT-95-17/1-T $\quad 110,585,721$

Prosecutor v Jokic IT-01-42-PT and IT-01-46-PT, 20.2.02 852

Prosecutor v Morris Kallon and Brima Buzzy Kamara, (ICTR)Decision on Challenge to Jurisdiction: Lomé Accord Amnesty, SCSL-2004-15-AR72(E) and SCSL-2004-16-AR72(E), (Appeals Chamber, 13.3.04 732

Prosecutor v Karadžić (ICTY), IT-95-5

Prosecutor v Allieu Kondewa, (ICTR)Decision on Lack of Jurisdiction/Abuse of Process: Amnesty Provided by the Lome' Accord, SCSL-04-14-T-128-7347 (Appeals Chamber, 25.5.04)

Prosecutor v Kordić and Cěrkez,(Judgment) ICTY-95-14/2-T

$\begin{array}{ll}\text { Prosecutor v Krajisňik, IT-00-39, Trial Chamber Judgment, 27.9.06 } & 215 \\ \text { Prosecutor v Krnojelac (ICTY-97-25), Trial Chamber, 15.3.02 } & 585\end{array}$

Prosecutor v Krstić, IT-98-33, Trial Chamber Judgment, 2.8.01; Appeals Chamber Judgment, 19.4.04 215

Prosecutor v Kunarac, IT-96-23\&23/1 Appeals Chamber, 20.6.02 573

Prosecutor v Kvocka, ICTY-98-30-T, 2.11.01 53

Prosecutor v Stakic Milomar, ICTY-2003-IT-97-24-T, (Judgment) (13.7.03) 739

Prosecutor v Milosevic ICTY $\quad 721$

Prosecutor v Naletilic and Martinovic (Judgment) ICTY-98-34-T 506

Prosecutor v Dragan Nikolic' (Decision on Interlocutory Appeal concerning
Legality of Arrest) ICTY-IT-94-2-AR73 (5.6.03) $\begin{array}{lr}\text { Legality of Arrest) ICTY-IT-94-2-AR73 (5.6.03) } & 740 \\ \text { utor v Simic et al, ICTY-95-9, Trial Chamber, 17.10.03 } & 585\end{array}$

$\begin{array}{lr}\text { Prosecutor v Simic et al, ICTY-95-9, Trial Chamber, 17.10.03 } & 585 \\ \text { Prosecutor v Tadić IT-95-1, 2.10.95 } & 278,427,496\end{array}$

Prosecutor v Savo Todovic ICTY-2006-IT-97-25/1-AR11bis 722

Provimi Ltd v Roche Products Ltd [2003] EWHC 961 (Comm) 556

Prudential Assurance v Newman Industries [1980] 2 WLR 339 396 


\section{Case Index}

Public Prosecutor v Apava Keru; Public Prosecutor v Aia Moroi [1985] PNGLR 78

Public Prosecutor v Silas [1989-1994] 2 Van LR 659

Pulp Mills on the River Uruguay [2006] ICJ Reports; [2007] ICJ Reports

Pusa Case C-224/02 [2004] ECR I-5763

151,721

1002

Pusi v Leni (Unreported, HC, Solomon Islands, cc 218/1995, 14.2.97)

Questions of Interpretation and Application of the 1971 Montreal Convention Arising from the Aerial Incident at Lockerbie [1992] ICJ Rep 114

Quinn v Ireland (2001) 33 EHRR 264

R (Al-Jedda) v Secretary of State for Defence [2007] UKHL 58, [2008] 1 AC 332

[2006] EWCA Civ 327, [2007] QB 621

R (Al-Saadoon and Mufdhi) v Secretary of State for Defence, [2008] EWHC 3098; [2009] EWCA Civ 7

R (Al-Skeini) v Secretary of State for Defence [2007] 1 AC $153 \quad 227,700,764$

R (B) v Secretary of State for Foreign and Commonwealth Affairs [2005] QB $643 \quad 706$

R (Bagdanavicius) v SSHD [2005] 2 WLR 1309 (HL) 540

$\mathrm{R}$ (Bancoult) v Secretary of State for Foreign and Commonwealth Affairs (No 2) [2008] 3 WLR 955

R (Binyam Mohamed) v Secretary of State for Foreign and Commonwealth Affairs [2008] EWHC 2048

R (Corner House Research) v Director of Serious Fraud Office [2008] 3 WLR 568

R (Countryside Alliance) v Attorney-General [2008] 2 All ER 95

R (European Roma Rights Centre) v Prague Immigration Officer [2005] 2 AC 1

R (Hurst) v Coroner for Northern District London [2007] 2 AC 189

$\mathrm{R}$ (Limbuela) v Secretary of State for the Home Department HL 3.11.05

R (National Association of Health Stores) v Department of Health [2005] EWCA Civ 154

R (Othman (Jordan)) v Secretary of State for the Home Department [2008] EWCA Civ 290

R (Peupushi) v CPS [2004] EHWC 798

R (Pretty) v Director of Public Prosecutions (18.10.01 October 2001, HL)

R (R) v Durham Constabulary and Another [2003] 3 All ER 419, [2005] UKHL 21

R (Razgar) v Secretary of State for the Home Department [2004] 3 WLR 58

$\mathrm{R}$ (Tesema) v Secretary of State for the Home Department

R (Ullah) v Special Adjudicator [2004] 2 AC 323

R ex p Zeqiri v Secretary of State for the Home Department [2002] (HL)

R v B (SA) [2003] 2 SCR 678

R v Bow Street Magistrates Court, ex parte Pinochet Ugarte (No. 3) [1998] 4 All ER 897

R v Bow Street Metropolitan Stipendiary Magistrate; Ex parte Pinochet (No 3) [2000] 1 AC 14

R. v Brent London Borough Council, ex p. Gunning (1985) 84 LGR 168

R v Chief Immigration Officer; Ex parte Salamat Bibi [1976] 1 WLR 979

R v Devon County Council, ex p Baker (1993) COD 138

R v Director of Public Prosecutions; Ex parte Kebilene [2000] 2 AC 326

R v Gilbert (1977) 66 Cr App R 237

R v H [1994] 2 NZLR 143

R v Hape [2007] 2 SCR 292

Rv HM Treasury and Commissioners of Inland Revenue, ex parte Daily Mail and General Trust plc, ECJ Case 81/87, [1988] ECR 5483

465

453, 758

986

450

453

529

467

708

453

535

855

529

529

705, 761

529

844

110

467, 694

373

453

373

453

857

39

462

1022

529

453

R v Khan [1997] AC 558

R v Liverpool Corporation, ex p Liverpool Taxi Fleet Operators' Association (1972) 2 QB 299

R v Loumia and Others [1984] SILR 51 


\section{International and Comparative Law Quarterly}

R v Secretary of State for the Home Department ex parte Adan [1999] INLR 362

R v Secretary of State for Home Department; Ex parte Adan [2001] 2 AC 477

R v Secretary of State for the Home Department ex parte Aitseguer [2001] 2 WLR $143 \quad 529$

R v Secretary of State for the Home Department Ex parte Ali [2000] Imm AR $134 \quad 453$

R v Secretary of State for the Home Department Ex parte Brind [1991] 1 AC $696 \quad 452$

R (Horvath) v Secretary of State for Environment, Food and Rural Affairs ECJ Case C-428/07, 16.7.09

R v Secretary of State for Home Department; Ex parte Kirkwood [1984] 1 WLR 913

R v Secretary of State for Home Department; Ex parte Launde [1997] 1 WLR $839 \quad 453$

R v Secretary of State for Home Department; Ex parte Pierson [1998] AC 539

R v Secretary of State for Home Department; Ex parte Simms [2000] 2 AC 115

R v Secretary of State for Home Department; Ex parte Stafford [1999] 2 AC $38 \quad 462$

R v Secretary of State for Home Department; Ex parte Thakrar [1974] QB $684 \quad 460$

R v Secretary of State for Home Department Ex parte Yemoh [1988] Imm A R $595 \quad 449$

R v Shaw [1991] 8 CRNZ 511

Rahimtoola v Nizam of Hyperbad [1957] 3 All ER 441

Rainbow Warrior, Decision of 30.4.90, UNRIAA, Vol. XX 274

In the matter of sentencing Taha Yassin Ramadan, Application for Leave to Intervene as Amicus Curiae and Application in Intervention as Amicus Curiae of UNHCR, Iraqi High Tribunal, 8.2.07

Rasul v Bush, 542 US 466 (2004)

Rathwell v Rathwell [1978] 2 SCR 436

RD (Algeria) [2007] UKAIT 00066

Re Astor's Settlement Trusts [1952] Ch 534

Re B, R v Special Adjudicator ex parte Hoxha, 2005

Re Chong Bing Keung (No 2) [2000] 2 HKLRD 571

Re Chung Tu Quan [1995] 1 HKC 566

Re the Constitution, Attorney General v Olomalu [1980-93] WSLR 41

Re the Constitution, Taamale v Attorney-General (Unreported, SC, Samoa, 1995)

Re the Constitution, Timakata v Attorney-General [1992] VUSC 9, (1980-1994] Van LR 691

In re G (Adoption: Unmarried Couple) [2009] 1 AC 173

Re Londonderry's Settlement [1965] Ch 918

Re Minister for Immigration and Multicultural Affairs; Ex parte Lam (2003) 214 CLR 132

Re Pham Van Ngo [1991] 1 HKLR 499

Re: Piracy Jure Gentium [1934] AC 586

Re a Shop Fitting Contract [1993] ILPr 395

Re VCE v FCT [2006] AATA 821

Re Miriam Willingal (1997) 2 CHRLD 57

Reference by the Queen's Representative [1985] LRC (Const) 56

Reference by Western Highlands Provincial Executive (unreported, SCJ, PNG, 20.9.95)

Reineccius v Bank of International Settlements, Partial Award (PCA, 2002)

Request for Interpretation of the Judgment of 31.3.04 in the Case Concerning Avena and Other Mexican Nationals (Mexico v USA), Judgment 19.1.09

Retail, Wholesale \& Department Store Union, Local 580 et al v Dolphin Delivery Ltd (1986) 33 DLR (4th) 174

Rewe-Zentrale AG v Bundesmonopolverwaltung für Branntwein, Case 120/78, [1979] ECR 649

Right to Information on Consular Assistance in the Framework of Guarantees for Due Legal Process, Advisory Opinion OC-16/99, IACHR, Series A 16(1999) 980

Roche Nederland ECJ Case C-539/03 13.7.06

Royal Bank of Scotland, C-488/07, [2009] ECR I-000 
Saghi v Islamic Republic of Iran Award No 544-298-2, 29 Iran-U.S.C.T.R. 20 (1993) 139

Salduz v Turkey ECtHR Appl. no 36391/02, 27.11.08

Salman v Turkey (2002) 34 EHRR 17

Saluka Investments BV v Czech Republic, Swiss Fed. Ct, 17.3.06

Samoan Public Trustee v Collins [1961] WSNZCA 1

Sandline International v Papua New Guinea (1998) 117 ILR 552

Saramaka People v Suriname, IACHR, Series C 172 (2007)

Sarikaya v Turkey, Appl. No 36115/97, 22.4.04

Saunders v UK (1997) 23 EHRR 313

Sawhoyamaxa Indigenous Community v Paraguay, IACHR, Series C 146 (2006)

A.L.A. Schechter Poultry Co. v US (1935) 295 US 495

Schempp Case C-403/03 [2005] ECR I-6421

Schmidberger Case C-112/00 [2003] ECR I-5659

Schmidt v Rosewood [2003] UKPC 26; [2003] 2 AC 709

SDC, C-2/95 [1997] ECR I-3017

Secretary for Security v Prabakar [2005] 1 HKLRD 289

Secretary of State for the Home Department v K

Secretary of State for the Home Department v MB [2008] AC 440

858

581

303

182

464

980

858

837

980

358

1003

989

661

901

447

529

763

Secretary of State for the Home Department, ex p Thangarasa \& Yogathas, [2002] $36 \mathrm{HL}$

Sefo v The Attorney-General and the Alii and Faipule of Saipipi (SC, Samoa, 12.7.00), [2000] WSSC 18

Sellers v Maritime Safety Inspector [1999] 2 NZLR 44

Selmouni v France (2000) 29 EHRR 403

Sepet v Secretary of State for the Home Department [2003] 1 WLR 856

Serves v France (1999) 28 EHRR 265

SEVIC Systems AG, C-411/03, [2005] ECR I-10805

Shamayev v Georgia and Russia ECHR 2005-III

Sheekh v Netherlands (2007) 45 EHRR 50

Shergold v Tanner (2002) 209 CLR 126

Shevill, C-68/93, [1995] ECR I-415

Shogun Finance v Hudson[2003] UKHL 62, [2004] 1 AC 919

Simmenthal, ECJ Case 106/77, 9.3.78

Skatteverket v AB SKF, ECJ Case C-29/08

Skidmore Energy, Inc et al v KPMG et al No. 3:03-CV-2138 (N.D. Tex. 3.12.04)

SKF [2009] ECR 1-000

Soering v UK (1989) 11 EHRR 439

Solicitor (24/07) v Law Society of Hong Kong [2008] 2 HKLRD 576

Solleveld, C-443/04, [2006] ECR I-3617

Solomou and Others v Turkey, ECtHR Appl. No. 36832/97

Sorochan v Sorochan [1986] 2 SCR 38

South Dakota v Department of Interior (1995) 69 F3d 878 (8th Cir)

South-West Africa, International Status, [1950] ICJ Reports 140

Southern Pacific Properties (Middle East) v Egypt (1992) Award ICSID Case No ARB/84/3, Award (20.5.92)

Starrett Housing Corp v Islamic Republic of Iran, Award No 314-24-1, 16 Iran-U.S.C.T.R. 112, 202 (1987)

State of Connecticut, et al v American Electric Power Company, et al (2005) 406 F Supp 2d. 265 (SDNY) (under appeal)

Stiching Uitvoering Financiële Acties, Case 348/87, [1989] ECR 1737

Stock v Frank Jones (Tipton) Ltd [1978] 1 WLR 231

Stockholm Lindopark, C-150/99, [2001] ECR I-493

Sumpter v Hedges [1898] 1 QB 673

604

902

35

902

875

571

357

359

450 


\section{International and Comparative Law Quarterly}

T v Secretary of State for the Home Department [1996] Imm AR 443

Ta'amale and Ta'amale Toelau v The Attorney-General (CA, Samoa, 18.8.95), [1995] WSCA 12

Tachiona v Mugabe (2001) 169 F Supp 2d 259

Tadič, ICTY Appeals Chamber, 2.10.95

Tam Hing-yee v Wu Tai-wai [1992] 1 HKLR 185

Tam Nga Yin v Director of Immigration (2001) 4 HKCFAR 251 456

Tamplin v James (1880) 15 Ch D 215

Tan Te Lam v Superintendent of Tai A Chau Detention Centre [1997] AC $97 \quad 445$

Tang Ping-Hoi v Attorney-General [1987] HKLR 324

Tarbert Trading, Ltd v Cometals, Inc, 663 F. Supp. 561

Tas-Hagen, C-192/05 [2006] ECR I-10451 1003

Tataki, Michael Xysters, Human Rights Application [1996] PNGLR $90 \quad 40$

Tavita v Minister of Immigration [1994] 2 NZLR 257

Teitinnang v Ariong [1987] LRC (Const) 517

Telfner v Austria (2002) 34 EHRR 207

Telstra Corp v Worthing (1999) 197 CLR 61 461

Temple of Preah Vihear (Cambodia v Thailand) [1961] ICJ Rep 31, [1962] ICJ Rep 6

Teonea v Pule o Kaupule and Nanumaga Falekaupule (HC, Tuvalu, 11.10.05), [2005] TVHC 2

Test Claimants in Class IV of the ACT Group Litigation v Commissioners of Inland Revenue, ECJ C-374/05, 12.12.06

$\begin{array}{lr}\text { Thor Shipping A/S v The Ship 'Al Duhail' [2008] FCA 1842 } & 689 \\ \text { Thornhill v Attorney General for Trinidad and Tobago [1981] AC 61 } & 47\end{array}$

Tierce Ladbroke and Derby, Joint Cases C-231/07and C-232/07, [2008] ECR 1-73 903

Tin Council Case [1990] AC 418

Tokai Carbon and others v Commission T-236/01, T-239/01, T-244/01, T-251/01 and T-252/01 [2004] ECR II-1181

Tomimaru (Japan v Russian Federation) (Prompt Release, Judgment of 6.8.07)

Touby v US (1991) 111 Sup Ct, 1752

Tower Taxi Technology LLP v Marsden \& ors [2005] EWHC 1084, [2005] EWCA Civ 1503

Transporti Castelletti Spedizioni Internazionali SpA v Hugo Trumpy SpA, ECJ Case C-159/97, [1999] ECR 1597

Trendtex Trading Corp v Central Bank of Nigeria [1977] 1 QB 529 Republic of Guyana [2008] CCJ 1 (OJ) $\quad 683$

Tuivaiti v Faamalaga [1980-93] WSLR 19

Turkey-Textiles, WTO Appellate Body Report WT/DS34/AB/R
Überseering BV v Nordic Construction Company Baumanagement $\mathrm{GmbH}$, C-208/00, ECR [2002] I-9919

Ulufa'alu v Attorney General [2005] 1 LRC 698

Unión de Pequeños Agricultores v Council, C-50/00 P, [2002] ECR I-6677

United Kingdom v Iran (BP Case) (1974) 53 ILR 297

United States Diplomatic and Consular Staff in Tehran [1980] ICJ Reports 153, 497

United States v Booker 124 US Supreme Court 2531, 12.1.05

USA v Michael D Andreas and Terrance S Wilson (2000) No 96 CR 762 USCA 7 th District 


\section{Case Index}

Unterpertinger, ECJ C-212/01, [2003] ECR I-13859

Van Colle v Chief Constable of the Hertfordshire Police [2009] 1 AC 224

Van Gend \& Loos, ECJ Case 26/62, 5.2.63

Van Uden Maritime BV v Kommanditgesellschaft in Firma Deco-Line, C-391/95, ECJ I, 7091

Velasquez Rodriquez (1988) Inter-Am Ct HR 1

Verizon Communications Inc v Law Offices of Curtis V Trinko LLP, 124 S Ct $872 \quad 627$

Viking Line Case C-438/05 [2007] ECR I-10779

989

Vodafone, O2 et al ECJ Pending Case C-58/08

991

Volga (Russian Federation v Australia) (Prompt Release, Judgment of 23.12.02) ITLOS Rep, 200210

Volker Ludwig, C-453/05, [2007] ECR I-5083

Wagner v Radke (SC, Samoa, 19.2.97), [1997] WSSC 2

Walker v The York and Midland Railway Company, 2 E. \& B. 750

Waterschap Zeeuws Vlaanderen, C-378/02 [2005] ECR I-4685

Wayman v Southard (1825) 23 US, 1

Weiss v Austria, 15.5.03, UNHRC Communication No 1086/2002

358

709

West Rand Central Gold Mining Company v King [1905] 2 KB 391

Western Australia v Commonwealth (1995) 183 CLR 373

461

Weyhermüller, Krystyna Zabłocka v Land Baden-Württemberg, 4.12.08 1002

Wi Parata v Bishop of Wellington 3 NZ. Jur (N.S.) SC 72

Wiener v US (1958) 57 US 349

Winfat Enterprise (Hong Kong) Co Ltd v Attorney-General (HK) [1985] AC 733

360

World wide Volkswagen Cor. v Woodson (1980) 444 US 286

445

480

X (Minors) v Bedfordshire County Council [1995] 2 AC 633

$\begin{array}{ll}\mathrm{X} \text { and Y } \mathrm{v} \text { the Netherlands [1985] ECHR } 4 & 570\end{array}$

Yakus v US (1944) 321 US 414

Ygeia, ECJ Joint cases C-394/04 and C-395/04, [2005] ECR I-10373 902

Yusuf and Al Barakaat International Foundation v Council, ECJ Case T-306/01, 21.9.05 230

Z v UK (2002) 34 EHRR 3

Z and T v UK Appl. No. 27034/05, 28.2.06

591

760

Zimbabwe Lawyers for Human Rights v President of Zimbabwe, S-12-03; Civ. App. 311/99

\section{Austrian Courts}

Austrian Supreme Court, OGH 12.2.98, 2 Ob 328/97t http://cisgw3.law.npace.edu/cases/980212a3

Austrian Constitutional Court:

VfSlg 6783/1972

VfSlg 7653/1975

VfSlg 7791/1976

VfSlg 11669/1988

VfSlg 11.829/1988

VfSlg 11.756/1988

VfSlg 11.829/1988

VfSlg 11.916/1988

\section{French Courts}

Conseil d'Etat, Agyepong, Appl. 112842, 2.12.94, $\quad 528$

Conseil d'Etat, Ourbih, Appl. 171858, 23.6.97 528

Conseil d'Etat, Appl 282275, 12.6.06 538

Conseil d'Etat Assemblé, Société Arcelor Atlantique et Lorraine et autres, Appl 287110, 8.2.07, 


\section{International and Comparative Law Quarterly}

Cass req, Apr 20, 1921, D.P. 1922, I, 181. (Tenant could withhold rent after owner refused to make repairs)

Cass com, Jan 30, 1979, D.S. 1979, Inf. rap. 317. (lessee of defective computer disks could not suspend performance)

Court of Appeal in Orléans, 6.5.03, SA les jolies céramiques sans kaolin v Sté Mridul Entreprises, Sté Trademark Tiles LTD, Journal du Droit International 200, 193

Court of Appeal in Paris, 4th division, Section A, 26.4.06 SA Normalu c/ SARL Acet, CCE 2006, comm. 106, obs. C. Caron

Court of Appeal in Paris, 14.6.06

482

Court of Appeal in Paris, 4th division, Section A, 6.6.07, JCP 2007.II.10151 482

Court of Appeal in Paris, 4th division, Section B, 9.11.07, D. 2008. AJ 8

Court of Appeal in Paris, 4th division, Section A, 18.6.08, JCP 2008.II.10180 482

Court of Appeal in Versailles, 2.11.06, M-E Ancel, CCE 2008, no 1, chron 1, spec no 14

Court of Cassation, 1st Civil Division, 9.12.03, Cristal (patent infringement case) 477

Court of Cassation, Commercial Division, 20.3.07 (unfair competition case) 477

CRR (Commission de recours des réfugiés), SR (sections réunies):

Keklicekpinari, application no. 351244, 23.2.01

Sissoko, applications 361050 and 3730777.11 .01

Soumah, appl 368138, 7.12.01

Altun, appl 362645, 9.1.03

Wang, appls 404302 and 404411, 28.2.03

M, appl 423904, 17.10.03

Koffi Amani, appl 403498, 25.6.04

Nazia, appl 444000, 15.10.04

Tas, appl 489014, 4.3.05

Tabe, appl 519803, 29.7.05

Tribunal Administratif de Paris, Mohammad Afzali, No. 0712180/9/1, 9.8.07

TGI Paris, ord. Réf., 2.11.05

TGI Paris, ord. Réf. 11.10.06

\section{German Courts}

Bundesgerichtshof, Case VIII ZR 185/92 (9.3.94) 892

BGH 13.10.04, Hotel Maritime, IZR 163/02 481

BGH, 30.3.06, I ZR 24/03 481

BGH 15.2.07, I ZR 114/04 481

BGH, 17.9.08, III ZR 71/08 481

BVerfGE 7, $29 \quad 942$

BVerfGE 33, 125

BVerfGE 42, $20 \quad 942$

BVerfGE 61, $149 \quad 942$

BVerfGE 68, $319 \quad 942$

BVerfG, 2 BvR 1481/04 14.10.04 949

BVerfG, 2 BvR 2236/04, 30.6.09 948

Jorgić, Oberlandesgericht Düsseldorf, 4. Strafsenat 26.9.97 514

LG Köln, 20.4.01, 81 O 160/99, http://www.jurpc.de/rechtspr/20010148.htm 481

OLG Köln, 24.4.06, 6 U 145/05 481

OLG Köln, 30.10.07, 6W161/07 481

OLG Karlsruhe, 10.7.02 (Intel Fall) MMR 12/2002, $814 \quad 481$

Pfaff, BVerwG 2 WD 12.04, 21.6.05, http://www.bundesverwaltungsgericht.de 10

OGH, 29.5.01, 4 Ob 110/01g, BOSS, Ö JZ 2001, 848 481

OGH, 11.8.05, 4 Ob 98/ 481

VfGH 11.10.01, G 12/00 947

\section{International Chamber of Commerce}

ICC Case No 2583, (1976) VII Ybk Intl Arbitration 124

ICC Case no 3267 of 1979, Collection of ICC Arbitral Awards (vol 1 1974-1985) $76 \quad 894$

ICC Case no 3540 of 3.10.80, (1981) 4 Journal du droit international (Clunet) $914 \quad 893$ 


\section{Case Index}

ICC Case No 4629 (1993) XVIII Ybk Commercial Arbitration 11

ICC Case no 8547 of 1999 (2003) XXVIII Ybk Commercial Arbitration 27

ICC Case no 9448 of 1999 http://www.unilex.info $\quad 890$

ICC Case no 11849 of 2003 (2006) XXXI Ybk Commercial Arbitration 148

Israeli Courts

Supreme Court of Israel, sitting as the High Court of Justice, HC 5100/94 and others, 6.9 .99 


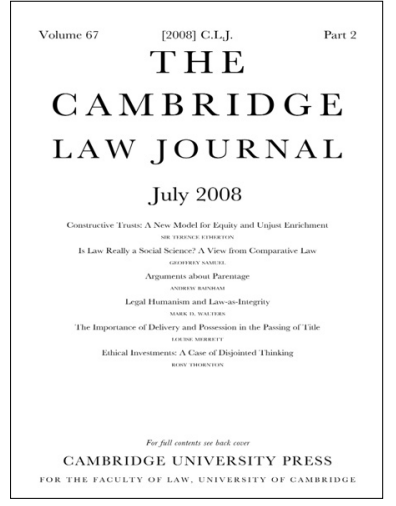

Cambridge Law Journal

is available online at

http://journals.cambridge.org/clj

To subscribe contact

Customer Services

in Cambridge:

Phone +44 (0)1223 326070

Fax +44 (0)1223 325150

Email journals@cambridge.org

in New York:

Phone (845) 3537500

Fax (845) 3534141

Email

subscriptions_newyork@cambridge.org

\section{THE \\ CAMBRIDGE \\ LAW JOURNAL}

Published for the Faculty of Law, University of Cambridge, UK

\section{Editor}

David J. Ibbetson, University of Cambridge, UK

The Cambridge Law Journal publishes articles on all aspects of law. Special emphasis is placed on contemporary developments, but the journal's range includes jurisprudence and legal history. An important feature is the Case and Comment section, in which judicial decisions, new legislation and law reform proposals are analyzed.

\section{Price information is available at http://journals.cambridge.org/clj}

\begin{tabular}{|c|}
\hline Free email alerts \\
Keep up-to-date with new material - sign up at \\
http://journals.cambridge.org/register
\end{tabular}

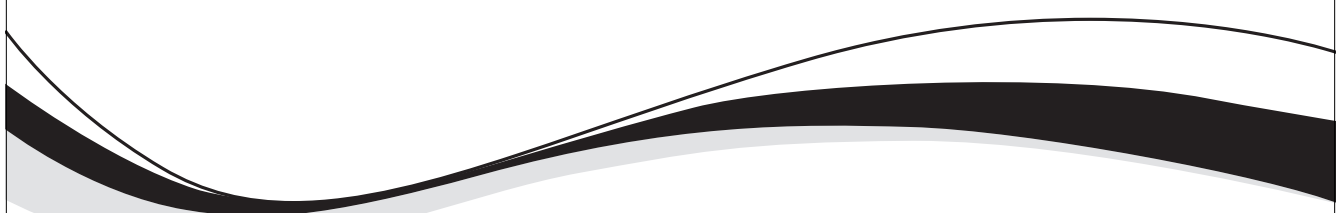




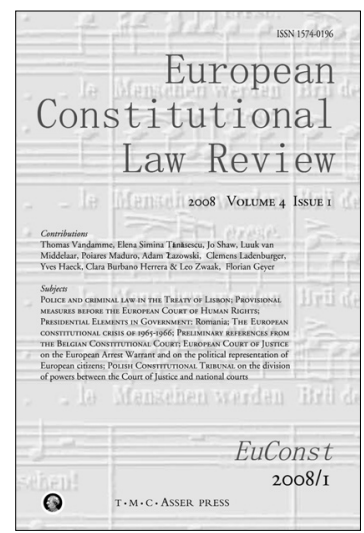

European Constitutional Law Review

is available online at

http://journals.cambridge.org/ed

To subscribe contact

Customer Services

in Cambridge:

Phone +44 (0)1223 326070

Fax $+44(0) 1223325150$

Email journals@cambridge.org

\section{in New York:}

Phone +1 (845) 3537500

Fax +1 (845) 3534141

Email

subscriptions_newyork@cambridge.org

\section{European \\ Constitutional \\ Law Review}

Marketed and distributed for T.M.C. Asser Press

\section{Editor-In-Chief}

W. T. Eijsbouts, University of Amsterdam, The Netherlands Marketed in distributed for T.M.C. Asser Press.

The European Constitutional Law Review, a peer reviewed English language journal, is a platform for advancing the study of European constitutional law, its history and evolution. Its scope is European law and constitutional law, history and theory, comparative law and jurisprudence. Published triannually, it contains articles on doctrine, scholarship and history, plus jurisprudence and book reviews.

\section{Price information is available at http://journals.cambridge.org/ed}

Free email alerts
Keep up-to-date with new material - sign up at
http://journals.cambridge.org/alerts

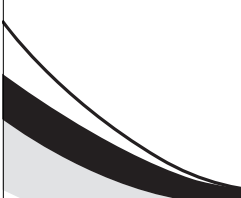

For free online content visit http://journals.cambridge.org/ed 


\title{
British Institute of International and Comparative Law
}

\author{
ADVISORY COUNCIL
}

Mr Dapo Akande

Judge David Anderson CMG

Sir Christopher Bellamy QC

Sir Franklin Berman KCMG QC

Mr Daniel Bethlehem QC

The Rt Hon Sir William Blair

Professor Alan Boyle

The Hon Sir Nicholas Bratza

Professor Ian Brownlie CBE QC

FBA DCL

The Rt Hon Lord Justice Carnwath

CVO

Ms Victoria Cochrane

Baron Collins of Mapesbury

Mr Tim Cowen

The Rt Hon Mr Justice Cranston

DCL QC

Professor James Crawford SC FBA

Ms Clare Dyer

The Rt Hon Sir David Edward

KCMG QC

Professor Piet Eeckhout

Professor Malcolm Evans

Ms Helen Fletcher Rogers

Lady Hazel Fox CMG QC

Dr David Freestone

Professor Sir Roy Goode CBE QC

LLD FBA

Professor Jonathan Harris

HE Judge Rosalyn Higgins

DBE QC
The Rt Hon Lord Hoffmann

The Rt Hon Lord Hope of Craighead

Mr Alan Jenkins

Judge Koen Lenaerts

Sir Elihu Lauterpacht CBE QC

Mr Justice Lloyd Jones

Mr Paul Lomas

Mr John Louth

The Rt Hon Chief Justice Beverley

McLachlin

The Rt Hon Lord Mance

HE Judge Thomas Mensah

The Rt Hon Lord Neill of Bladen QC

Sir Peter North CBE QC DCL FBA

The Rt Hon Lord Phillips of Worth

Maltravers

Lord Justice Richards

Professor Jonathan Rickford CBE

Mr Joshua Rozenberg

Judge Sir Konrad Schiemann

Lord Justice Sedley

Advocate-General Eleanor Sharpston QC

Professor Malcolm Shaw QC

Judge Bruno Simma

The Rt Hon Lord Slynn of Hadley

Judge Christiaan Timmermans

Mr Andrew Trollope QC

Mr Stephen Walzer

Professor Colin Warbrick

Dame Juliet Wheldon DCB QC 


\section{INTERNATIONAL \& COMPARATIVE \\ LAW \\ QUARTERLY}

\section{VOLUME 59}

\section{PART 1}

\section{JANUARY 2010}

\section{SHORTER ARTICLES}

The Jus Ad Bellum and Entities Short of Statehood in the

Report on the Conflict in Georgia

Christian Henderson and James A Green

Counter-Piracy Law Enforcement and Human Rights

Douglas Guilfoyle

CURRENT DEVELOPMENTS: PUBLIC INTERNATIONAL LAW

I. The Geneva Conventions and United Nations Personnel (Protocols)

Act 2009: A Move Away from the Minimalist Approach

Michael Mayer and Charles Garraway

II. Clearing the Fog of War? The ICRC's Interpretive Guidance on

Direct Participation in Hostilities

Dapo Akande

CURRENT DEVELOPMENTS: INTERNATIONAL COURTS AND TRIBUNALS

I. International Court of Justice Certain Questions of

Mutual Assistance in Criminal Matters (Djibouti v France)

Judgment of 4 June 2008

Robert Cryer and loannis Kalpouzos

II. The International Criminal Court Arrest Warrant Decision for

President Al Bashir of Sudan

Manisuli Ssenyonjo

BOOK REVIEWS

BOOKS RECEIVED

Cambridge Journals Online For further information about this journal please go to the journal website at: journals.cambridge.org/ICLQ

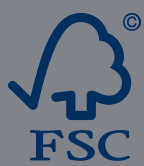

Mixed Sources

Product group from well-managed forests and other controlled sources www.fsc.org Cert no. T-COC-002769

(c) 1996 Forest Stewardship Council
CAMBRIDGE UNIVERSITY PRESS 